\title{
The development of IEC 61850 generic object oriented substation event monitoring application for Tenaga Nasional Berhad Smart Substation
}

\author{
M. S. Shokri ${ }^{a^{*}}$, M. I. Ridwan ${ }^{a}$, K. Zakaria ${ }^{a}$, R. M. Lajim ${ }^{a}$, A. Musa ${ }^{b}$ \\ ${ }^{a}$ TNB Research, Kawasan Institusi Penyelidikan, 43000 Kajang, Selangor, Malaysia \\ ${ }^{b}$ Tenaga Nasional Berhad, Brickfields, 50470 Kuala Lumpur, Malaysia
}

\begin{abstract}
Seamless information exchange in smart substations is vital to ensure the successful operation of functions in a smart grid domain. IEC 61850 is an international standard that enables interoperability between devices in smart substations through standardized data model and communication services. One of the communication services defined in IEC 61850 is the generic object oriented substation event (GOOSE). The communication using IEC 61850 GOOSE replaces point to point copper wiring between intelligent electronic devices (IED) in conventional substations with high speed communication over Ethernet technology. However, the implementation of GOOSE has posed new challenges, mainly in monitoring and managing signals that carry specific substation functions. This is because with GOOSE, these signals are transmitted using single Ethernet connection which carries significant amount of information in a form of network packets at any point of time. Therefore, there is a need for power utilities who implement IEC 61850 standard in substations, such as Tenaga Nasional Berhad (TNB) to develop tools which are able capable in administrating and maintaining the features of IEC 61850 in substations. This paper will discuss the development of a prototype IEC 61850 GOOSE monitoring application which is a part of TNB IEC 61850 Substation Intelligent and Management System (61850 SIMS). This paper will also elaborate on the application development process, which is according to the Software Development Life Cycle (SDLC). The application is designed to represent IEC 61850 GOOSE information in terms and expressions that can be appreciated by conventional protection and maintenance engineers. The prototype application was successfully developed and tested at TNB IEC 61850 System Verification and Simulation (SVS) laboratory. This paper will also discuss on the application capabilities and new features that are planned to be included for future improvements.
\end{abstract}

Keywords: Smart substation, IEC 61850, generic object oriented substation event (GOOSE)

\section{Introduction}

The term Smart Grid is commonly associated with modern electric power grid infrastructure that employs advanced information, communication and automation technologies. Reliable and real-time information becomes the key features in a smart grid environment [1]. The International Electrotechnical Committee (IEC) Strategic Group on Smart Grid (SG 3) identified an increased level of information sharing as one of the main points about smart grid [2]. In general, electrical substations are one of the main sources of information (e.g. device status for asset management, fault information for disturbance analysis). Therefore, it is imperative to deploy smart substations that support a high degree of information sharing. Smart substation is generally characterized by the full integration of electrical substation with information and communication infrastructure [4]. One of the main features of a smart substation is the utilization of substation information by an intelligent substation management system equipped with advanced computing and historian. The intelligent system is essential to perform intelligent monitoring

\footnotetext{
* Manuscript received July 10, 2013; revised July 30, 2013.

Corresponding author. Tel.: +60-19-251 0027; E-mail address: m.shahmi@tnbr.com.my..
} 
and automated analysis in order to promote better situational awareness compared to the traditional substation.

\subsection{IEC 61850}

The IEC Strategic Group on smart grid highlights that standardization plays a crucial role in providing the ability of information sharing which will be required to enable the development of new applications for a future power system [2]. The United States National Institute of Standards and Technologies (NIST) have defined IEC 61850 as one of the key standards for achieving interoperability in smart grid environment [3]. IEC 61850 is the first international communication standard for substation and utility automation. The three fundamental objectives of IEC 61850 are interoperability, free allocation of functions and future proof. The standard ensures interoperability between devices with predefined substation data models and communication services over open communication technologies. Interoperability provides substation multi-vendor intelligent devices the ability to freely communicate and exchange information with each other, and use the information for its functions [4].

\subsection{Tenaga Nasional Berhad (TNB) electricity technology roadmap}

For electricity industry in Malaysia, Tenaga Nasional Berhad (TNB) version of "smart grid" is characterized with the theme "smart and intelligent electricity delivery system". TNB Electricity Technology Roadmap (TRM) was developed to identify the routes and technology destinations for the application of new technology in TNB [4]. The implementation of smart substation is one of the key milestones in realizing reliable and self-healing power system under the "intelligent power delivery system" theme. From the utility perspective, IEC 61850 is the key enabler for achieving full substation automation and intelligent power delivery system [6].

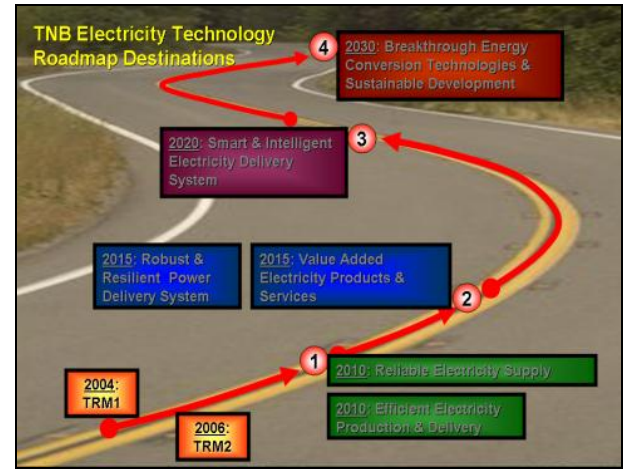

(a)

\section{TNB TECHNOLOGY ROADMAP}

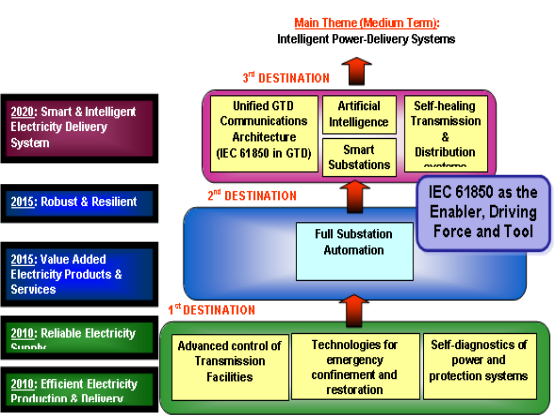

(b)

Fig. 1. TNB electricity technology roadmap (TRM): (a) general destinations and (b) detailed destinations.

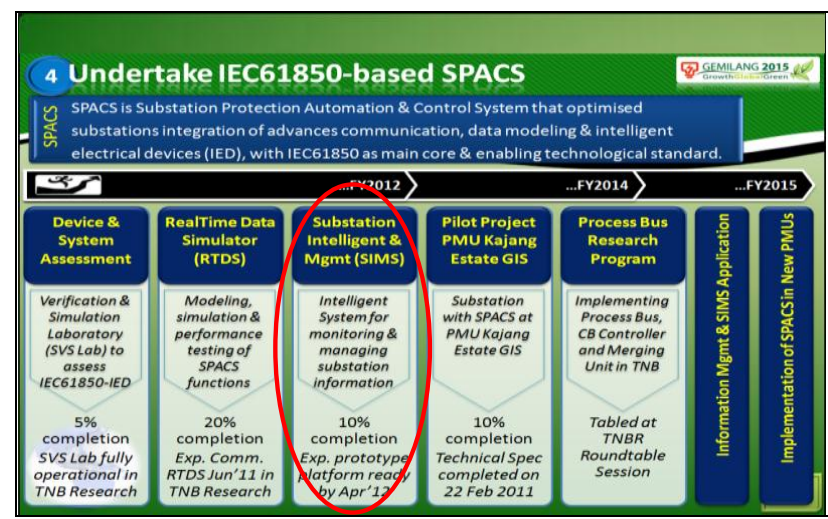

Fig. 2. Substation intelligent and management system (SIMS) as one of the seven key initiatives 
In order to achieve the identified TRM destinations (Fig. 1), TNB Transmission Division has defined several breakthrough key initiatives. One of the breakthrough key initiatives is to deploy proven technology, which undertakes IEC 61580 based Substation Protection, Automation and Control System (SPACS). The main objective of SPACS is to design next generation smart substations, with IEC 61850 standard as the main core and enabling standard. Seven key action plans have been identified to support the SPACS objective. One of the key action plans is the implementation of IEC 61850 Substation Intelligent and Management System (61850 SIMS), as shown in Fig. 2.

In April 2010, the Engineering Department of TNB Transmission Division has appointed TNB Research Sdn. Bhd. (TNBR), a wholly owned subsidiary of TNB, to carry out a joint two years Research and Development (R\&D) program entitled "Study and Development of Integrated and Standardized Engineering Workstation (EWS) Applications for TNB Transmission IEC 61850 Based Substation Automation System". As part of the project deliverables, a prototype of IEC 61850 Substation Intelligent and Management System (61850 SIMS) has been developed and tested in May 2012. The 61850 SIMS project was jointly developed between TNB and external system developer.

\subsection{IEC 61850 Substation Intelligent and Management System (61850 SIMS)}

The existing SIMS in TNB stands for Substation Interrogation and Monitoring System. It was developed mainly to ease interrogation of protection relays especially from remote. However, the current SIMS has several limitations. Station wide monitoring, automatic analysis and diagnostics becomes very difficult with the integration of various propriety software and communication protocols provided by multivendor Intelligent Electronic Device (IED) manufacturers.

The main purpose of the developed IEC 61850 Substation Intelligent and Management System (61850 SIMS) is to centralize the management and utilization of substation data and information from IEDs using IEC 61850 as the key enabler standard to support decision-making process, engineering, operation and maintenance, fault investigation and asset management [6]. The 61850 SIMS will be part of the overall architecture of a complete IEC 61850 based Substation Automation System (SAS), as shown in Fig. 3.

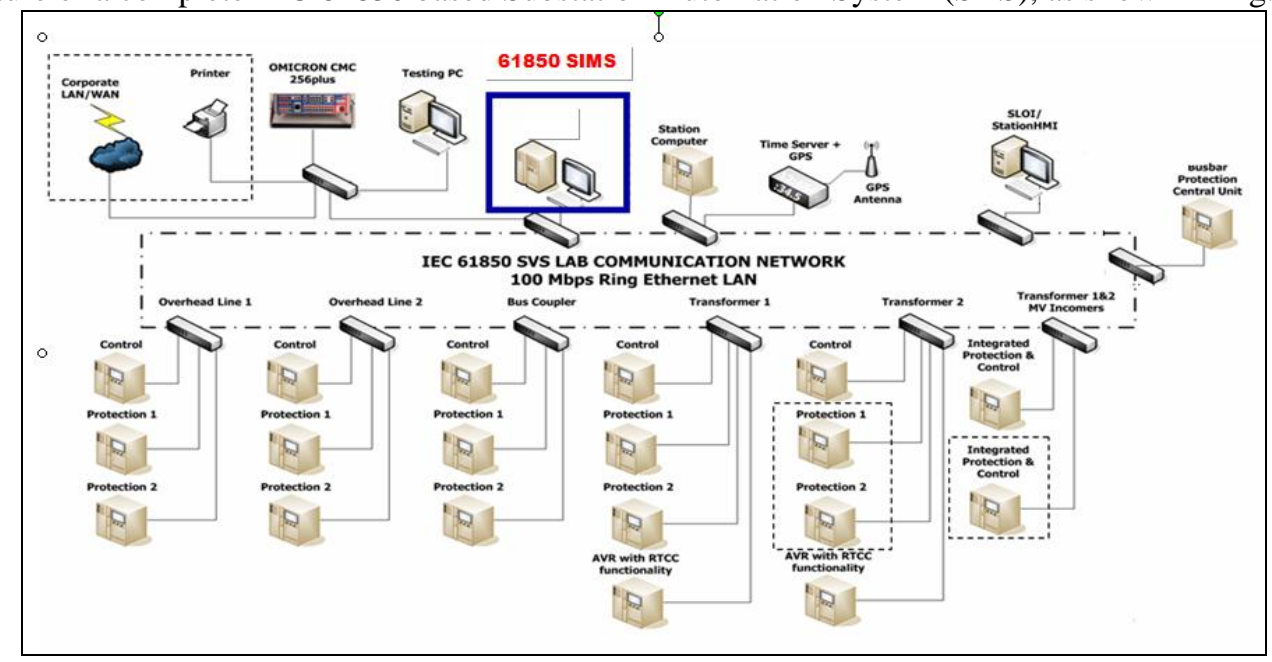

Fig. 3. IEC 61850 based substation automation system (SAS) architecture

The 61850 SIMS comprised of a total of 16 applications which are:

- IEC 61850 GOOSE Monitoring Application

- System Access User Interface

- Engineering Station Human Machine Interface (HMI)

- Substation Protection, Automation and Control (SPAC) System Management

- Communication Network Management

- Substation Interrogation and Monitoring Management for Proprietary and IEC 61850 based IED 
- COMTRADE Viewer

- Fault and Disturbance Analysis Viewer

- Substation Asset Monitoring

- Substation Configuration Management

- Substation Document Management

- Multi-Client Manager

- Database Management

- File Transfer

- Remote Access Interface

- Engineering Station Toolset

The next section will present one of the 61850 SIMS applications which is the IEC 61850 GOOSE Monitoring Application.

\section{IEC 61850 GOOSE Monitoring Application}

In a conventional power substation, hardwire connections are used to transmit signals between protection relays. However, the IEC 61850 GOOSE exploits Ethernet technology where the hardwire signals are substituted with Ethernet packets in a substation network. In order to maximize the availability and reliability of smart substation, it is crucial to monitor these GOOSE traffics. Network protocol analyzers such as Wireshark and Ethereal have the ability to decode and display GOOSE packets in a human readable form. However, these tools lack the features of interpreting GOOSE packets into meaningful information for protection and maintenance engineers. The vision of IEC 61850 GOOSE Monitoring Application is to utilize the parameters obtained from GOOSE packets and interprets them into meaningful information for troubleshooting and station wide maintenance purposes.

\subsection{Design and development}

The IEC 61850 GOOSE Monitoring Application is a part of a complex information system. Therefore, the concept of software development life cycle (SDLC) is applied to maintain a consistent methodology in the development process. The project goal and system requirements were initially compiled from the project proposal and other references such as TNB protection, automation and control specification. The end-user requirements were analyzed and documented as software requirement specification (SRS). The system design process was carried out according to the SRS. The approach of object-oriented design is chosen as a preference for the design process. Use case models and sequence diagram as shown in Fig. 4 and Fig. 5, were initially developed in the design phase.

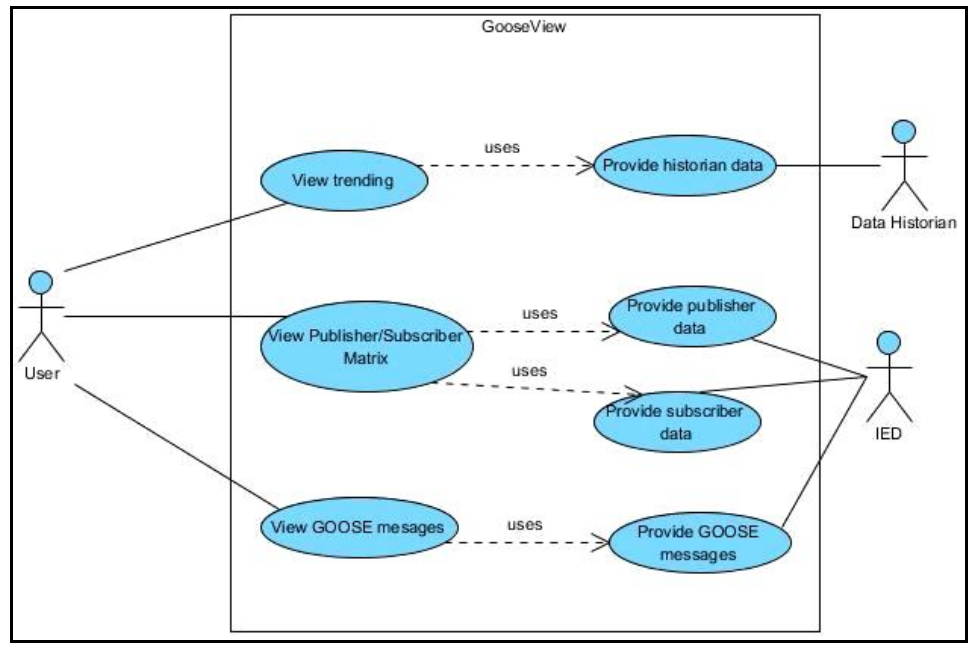

Fig. 4. Use case for IEC 61850 GOOSE monitoring application. 


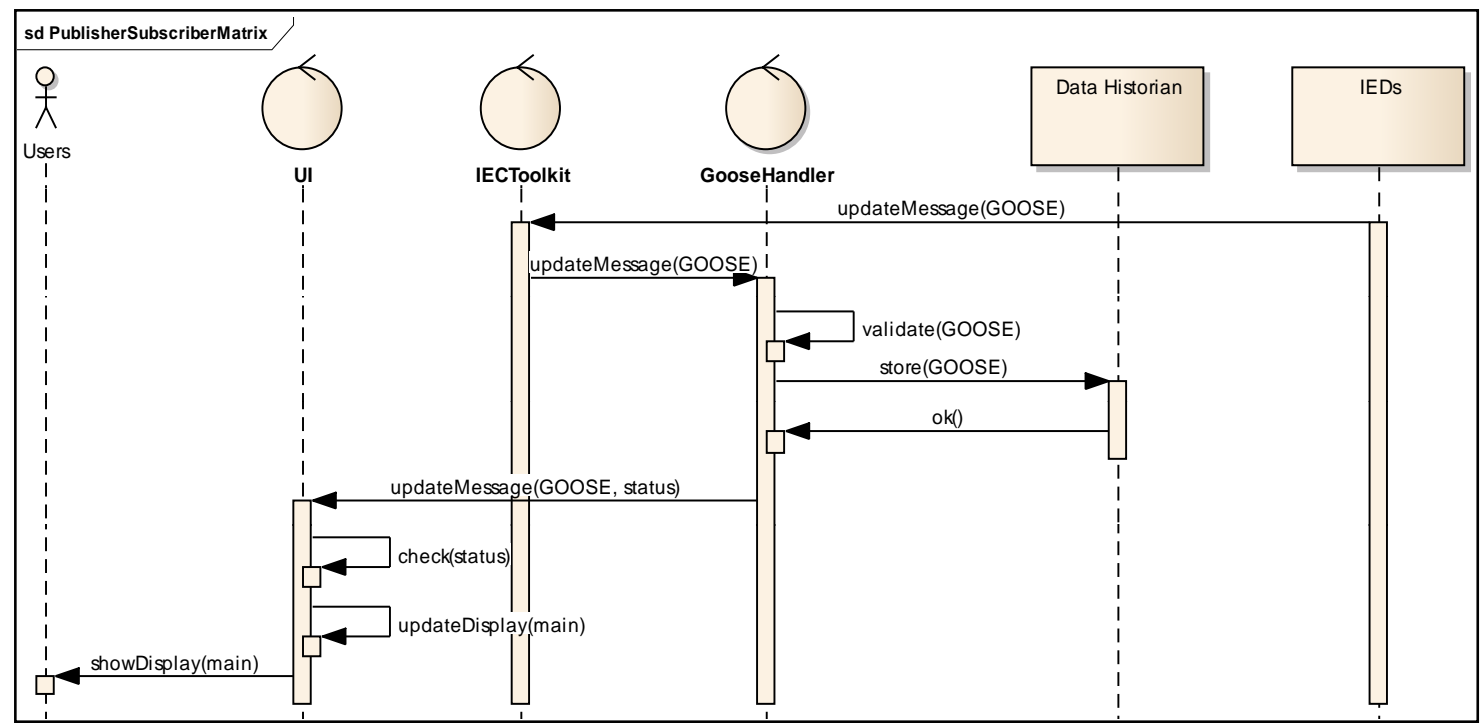

Fig. 5. Sequence diagram for IEC 61850 GOOSE monitoring application.

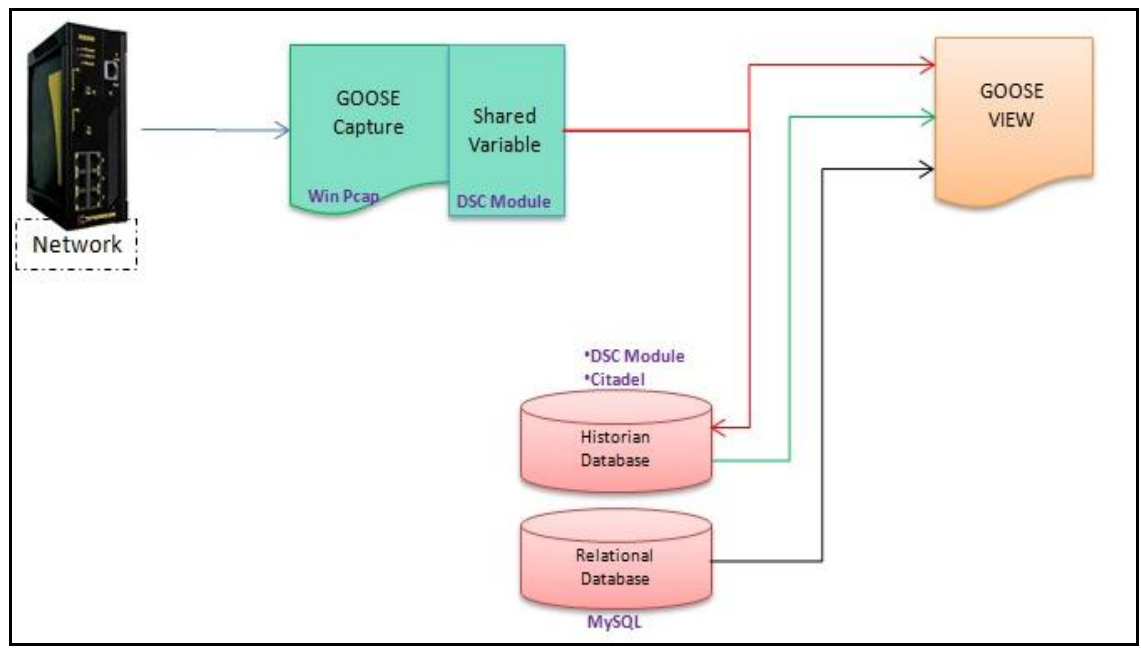

Fig. 6. Overview architecture for IEC 61850 GOOSE monitoring application

National Instuments LabVIEW ${ }^{\text {TM }}$ is chosen as the programming platform for the development of this application. The graphical programming method which is one of the main features of LabVIEW, enables simple source code management and straightforward detection of programming errors. The application utilizes 61850 SIMS database historians (National Instrument Citadel) to store historical GOOSE informationn, as shown in Fig. 6.

Windows packet capture (WinPCAP) libraries and GOOSE communication stack are the important components of GOOSE capture engine. The GOOSE communication stack was developed in-house according to IEC 61850 Part 8-1 and ASN.1 Binary Encoding Rules (BER). The main function of the stack is to decode and display GOOSE packets in human readable form. The GOOSE capture engine is designed to run in the background. Producer/consumer design pattern for events is chosen as the software design architecture for this application. This architecture facilitates GOOSE capture engine code to run asynchronously with the code that responds to Graphical User Interface (GUI) inputs without slowing down the responsiveness of the application. Fig. 7 displays the implementation of the producer/consumer design architecture for IEC 61850 GOOSE Monitoring Application. 


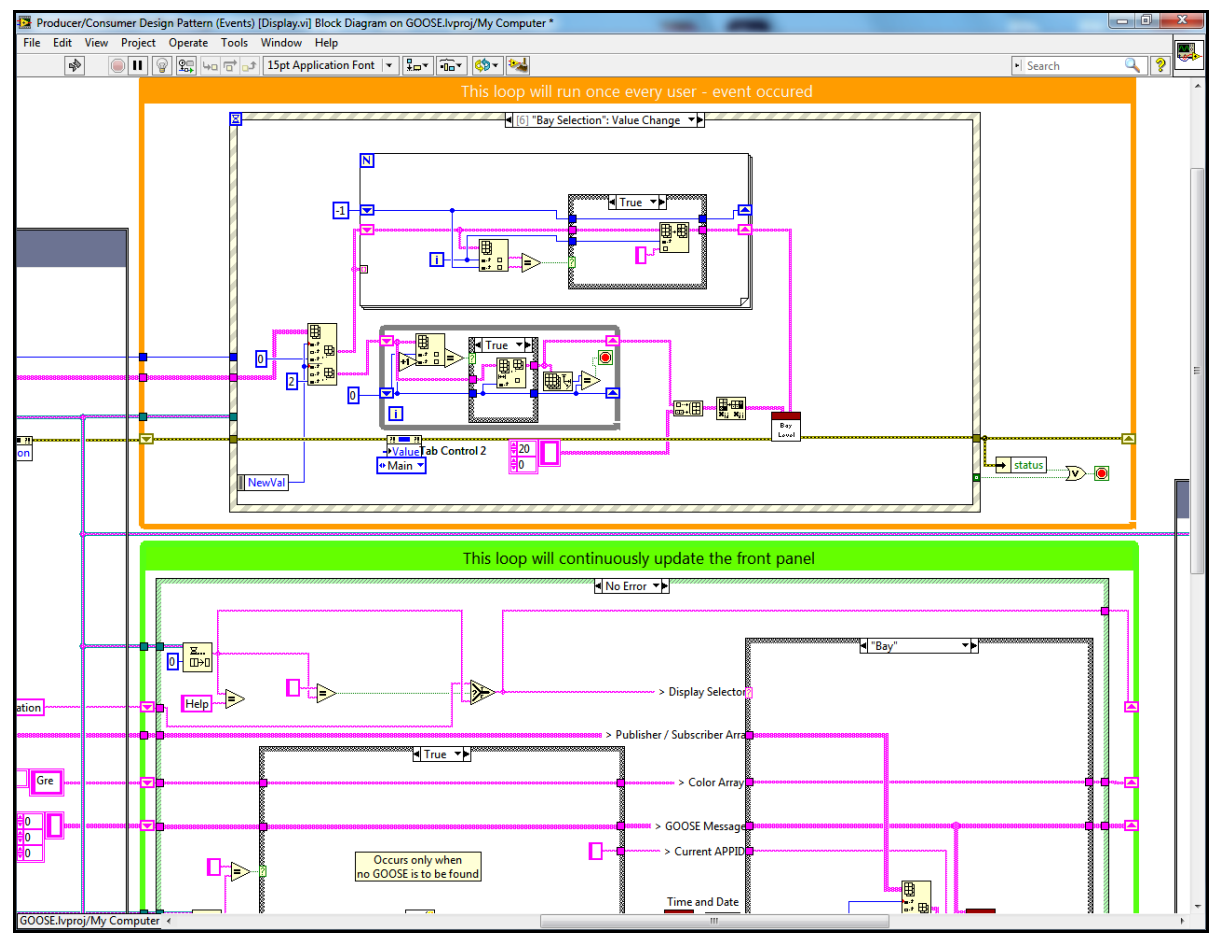

Fig. 7. The implementation of producer/consumer design pattern (events) in the source code of IEC 61850 GOOSE monitoring application.

\begin{tabular}{||l||l|c|l|c||}
\hline \multicolumn{1}{||l||}{ Type Of Data } & E01F11LP1 & E01F11LP2 & E01F34LC \\
\hline E03F11BCP1 & Breaker Failure Stage 2 & & & \\
\hline & SF6 Low Stage 2 & & & \\
\hline & 510 C Overcurrent Stage 1 & & & \\
\hline & Overvoltage Stage 2 & & & \\
\hline E03F11BCP2 & Breaker Failure Stage 2 & & & \\
\hline & External Fault Triggering & & & \\
\hline & CB Close Interlock & & & \\
\hline E03F34BCC & Circuit Breaker Q0 Status & & \\
\hline & Main Bus Isolator Q1 Status & & 0 \\
\hline & Reserve Bus Isolator Q2 Status & & 0 \\
\hline
\end{tabular}

Fig. 8. Detailed publisher-subscriber matrix.

\subsection{Features and capabilities}

IEC 61850 GOOSE Monitoring Application provides features that other tools (e.g. Wireshark, Ethereal, IEDScout) do not support. One of the features and capabilities is the display of GOOSE publisher subscriber matrix, as shown in Fig. 8. The GOOSE communication between IEDs can be clearly viewed through this matrix. The type of data in GOOSE packets are displayed in standardized terms that traditional protection and maintenance engineers can easily understand. For example, Circuit Breaker Q0 Status is displayed instead of XCBR\$ST\$Pos. The green indications show that there is a normal transmission of GOOSE packets from the publisher (the left column) to the subscribers (top right side of the matrix). If there is a data change in the GOOSE packets, the retransmission of GOOSE packets becomes faster. The application signifies these events with red indications.

IEC 61850 GOOSE Monitoring Application also provides a standardized platform to view and manage GOOSE information in the substation network. Fig. 9 shows a list of the GOOSE information on TNB SVS laboratory network that can be viewed using this application. 


\begin{tabular}{|c|c|c|c|c|}
\hline Publisher & goID & Type of Data & APPID & Subscriber \\
\hline E01F11LP1 & E01F11LP1_TRIP & Breaker Failure Stage 2 & 1 & BB Relay (Future) \\
\hline E01F11LP1 & E01F11LP1_START & AR Blocking & 2 & E01F11LP2 \\
\hline E01F11LP1 & E01F11LP1_START & Line Diff Comm Fail for zone 1 release & 2 & E01F11LP2 \\
\hline E01F11LP1 & E01F11LP1_START & Common External Fault Triggerring & 2 & E01F11LP2 \\
\hline E01F11LP1 & E01F11LP1_CTRL & $\mathrm{CB}$ Close Interlock to the $\mathrm{BCU}$ & 3 & E01F34LC \\
\hline E01F11LP1 & E01F11LP1_AR & 79 Initiate Phase A & 8 & E01F11LP2 \\
\hline E01F11LP1 & E01F11LP1_AR & 79 Initiate Phase B & 8 & E01F11LP2 \\
\hline E01F11LP1 & E01F11LP1_AR & 79 Initiate Phase $\mathrm{C}$ & 8 & E01F11LP2 \\
\hline E01F11LP1 & E01F11LP1_AR & Prepare 3P Trip & 8 & E01F11LP2 \\
\hline E01F11LP1 & E01F11LP1_AR & P1 Fail & 8 & E01F11LP2 \\
\hline E01F11LP2 & E01F11LP2_TRIP & Breaker Failure Stage 2 & 4 & Busbar Protection \\
\hline E01F11LP2 & E01F11LP2_TRIP & 79 Initiate Phase $\mathrm{A}$ & 4 & E01F11LP1 \\
\hline E01F11LP2 & E01F11LP2_TRIP & 79 Initiate Phase B & 4 & E01F11LP1 \\
\hline E01F11LP2 & E01F11LP2_TRIP & 79 Initiate Phase $\mathrm{C}$ & 4 & E01F11LP1 \\
\hline E01F11LP2 & E01F11LP2_TRIP & Prepare 3P Trip & 4 & E01F11LP1 \\
\hline E01F11LP2 & E01F11LP2_TRIP & P2 Fail & 4 & E01F11LP1 \\
\hline E01F11LP2 & E01F11LP2_START & AR Blocking & 5 & E01F11LP1 \\
\hline E01F11LP2 & E01F11LP2_START & Line Diff Comm Fail for zone 1 release & 5 & E01F11LP1 \\
\hline E01F11LP2 & E01F11LP2_START & Common External Fault Triggerring & 5 & E01F11LP1 \\
\hline E01F11LP2 & E01F11LP2_CTRL & $\mathrm{CB}$ Close Interlock to the $\mathrm{BCU}$ & 6 & E01F11LP1 \\
\hline E01F34LC & E01F34LC_ITL & Circuit Breaker Q0 Status & 7 & - \\
\hline E01F34LC & E01F34LC_ITL & Main Bus Isolator Q1 Status & 7 & E03F34BCC \\
\hline E01F34LC & E01F34LC_ITL & Reserve Bus Isolator Q2 Status & 7 & E03F34BCC \\
\hline E01F34LC & E01F34LC_ITL & Line Isolator Q9 Status & 7 & - \\
\hline E01F34LC & E01F34LC_ITL & Earth Switch Isolator Q8 Status & 7 & - \\
\hline E01F34LC & E01F34LC_ITL & Spare Isolator Status 1 & 7 & - \\
\hline E01F34LC & E01F34LC_ITL & Spare Isolator Status 2 & 7 & - \\
\hline E01F34LC & E01F34LC_ITL & Spare Isolator Status 3 & 7 & - \\
\hline
\end{tabular}

Fig. 9. GOOSE Information Management

\subsection{Future works}

Development is still in progress to improve the prototype IEC 61850 GOOSE Monitoring Application. The application will be integrated with new features. One of the new features is a sequence of event that interprets GOOSE parameter changes into an event list. The goal is to represent the information in an approach that can be appreciated by TNB protection and maintenance engineers. An example graphical user interface of the sequence of event list is shown in Fig. 10.

\begin{tabular}{cll}
\hline \hline Time & GOOSE ID & Events \\
\hline 12:00:01 & E01F11LP1_AR & is absent \\
12:00:12 & E01F11LP2_TRIP & in test mode \\
12:00:19 & E01F11LP1_START & needs commisioning \\
12:00:46 & E01F34LC_ITL & data change (T) \\
12:01:01 & Omicron_GOOSE & is new/additional GOOSE \\
12:01:05 & E01F11LP2_CTRL & new configuration revision (2) \\
\hline \hline
\end{tabular}

Fig. 10. Sequence of event for GOOSE monitoring application

\section{Conclusions}

As the public electric utility company in Malaysia, Tenaga Nasional Berhad (TNB) is fully aware of the potential and benefits of IEC 61850 standard in a smart substation environment. Realization of a appropriate substation monitoring tools or application is imperative in order to maximize substation availability and reliability. For a start, TNB have developed a prototype of IEC 61850 GOOSE Monitoring Application as a platform for more advance and intelligent monitoring of GOOSE traffic within the network of smart substation. This application itself is an essential medium to gain user acceptance and appreciation for the future implementation of smart substation in TNB. 


\section{Acknowledgements}

Acknowledgement is made to TNB Transmission and to all relevant people who have contributed to the success of the project. The author would also like to take this opportunity to express gratitude to the Management of TNB Research Sdn. Bhd. Especially the Managing Director, General Manager (T\&D) and Project Director/Senior Manager, who have supported the project team during the implementation of this research and development project.

The authors would also like to thank all individuals involved in this project, for their contributions, inputs, comments and moral support in completing the project. Special appreciation dedicated to TNB Transmission, especially Engineering Department and project collaborators, Matrix Power Network Sdn. Bhd., National Instruments (USA) and Virtual Instruments (Malaysia) for their contribution in making the project successful.

\section{References}

[1] Gungor VC, Buccella C, Hancke GP. Smart grid technologies: communication technologies and standard. Presented at: IEEE Transaction on Industrial Informatics 2011.

[2] IEC Smart Grid Standardization Roadmap. Edition 1.0 (2010).

[3] NIST Framework and Roadmap for Smart Grid Interoperability Standards, Release 1.0. NIST Special Publication 1108 (2010).

[4] Musa A. IEC 61850, the enabler of smart substation. Presented at: TNB ICT Technical Conference 2011.

[5] Ridwan MI, Zarmani MH, Lajim RM, Musa A. TNB IEC 61850 system verification and simulation (SVS) laboratory: Enabler to a successful smart grid implementation. Presented at: IEEE Innovative Smart Grid Technologies - Asia (ISGT Asia), 2012.

[6] Lajim RM, Hashim MF, Shokri MS, Zakaria K, Shaii AQ. Study and development of integrated and standardized engineering workstation (EWS) applications for TNB transmission IEC 61850 based substation automation system. Research Report. TNB Research Sdn. Bhd., 2012. 